\title{
Analysis on the Utility and Implementation Path of Copyright Insurance Based on Blockchain
}

\author{
Yiding Li \\ Business School, China University of Political Science and Law, Beijing, China \\ Email: liyiding@eximbank.govm.cn
}

How to cite this paper: Li, Y. D. (2021). Analysis on the Utility and Implementation Path of Copyright Insurance Based on Blockchain. Open Journal of Social Sciences, 9, 543-556.

https://doi.org/10.4236/jss.2021.97039

Received: June 25, 2021

Accepted: July 27, 2021

Published: July 30, 2021

Copyright $\odot 2021$ by author(s) and Scientific Research Publishing Inc. This work is licensed under the Creative Commons Attribution International License (CC BY 4.0).

http://creativecommons.org/licenses/by/4.0/

\section{(c) (i) Open Access}

\begin{abstract}
With the popularization and development of information networks, a large number of infringements have become constraints on online copyrights. By virtue of its compensation function and system advantages, copyright insurance can have a positive effect on online copyright. Based on the blockchain technology, this paper systematically elaborates the specific measures of the blockchain to solve the copyright insurance dilemma, and proposes the idea of building a copyright insurance platform module to realize the automatic claim settlement function of insurance from the system. However, technological changes have brought development opportunities as well as challenges. These challenges need to be gradually overcome in future development.
\end{abstract}

\section{Keywords}

Copyright Insurance, Blockchain, Risk Pricing, Insurance Claims, Automatic Execution

\section{Introduction}

With the rapid development of the digital economy, the comprehensive construction of a digital economy with data as a key element, and the continuous expansion of the digital extension of works, have accelerated the integration of core copyright industries such as news and publishing, broadcasting, film and television, animation and games, and Internet technology. These works express the copyright owner's thoughts in the form of codes with the help of computer language, forming network copyright (Cao, 2017). A large number of works under network copyright have problems such as uncertain ownership, inability to register copyright, and lack of a unified network copyright management plat- 
form, which has caused network copyright to become a low-cost infringement object. Copyright insurance under blockchain technology can provide a new way to protect network copyright. Based on the blockchain technology, this paper systematically elaborates the specific measures of the blockchain to solve the copyright insurance dilemma, and proposes the idea of building a copyright insurance platform module to realize the automatic claim settlement function of insurance from the system. However, technological changes have brought development opportunities as well as challenges. These challenges need to be overcome in future development (China Academy of Information and Communications Technology, 2018).

\section{The Concept and Characteristics of Blockchain Technology}

In order to analyze the effectiveness of copyright insurance based on blockchain in a scientific, comprehensive and efficient manner, this article first briefly elaborates the definition, concepts, and characteristics of blockchain technology in order to facilitate the subsequent research and analysis.

\subsection{Definition of Blockchain Technology}

In 2016, the Ministry of Industry and Information Technology of my country issued the "White Paper on China's Blockchain Technology and Application Development", which clearly defined the blockchain (Cui, 2019). Blockchain is a new application mode of computer technology such as distributed data storage, point-to-point transmission, consensus mechanism, and encryption algorithm. Among them, distributed is relative to centralized. In the white paper, distributed is one of the typical characteristics of the blockchain. It is a model that does not rely on a central server and uses distributed computer resources to perform calculations; the consensus mechanism is to achieve trust and acquisition between different nodes in the blockchain system. The mathematical algorithm of equity; a smart contract is a contract that uses computer language instead of legal language to record terms. In layman's terms, the blockchain is like a huge public ledger, which is kept and maintained by all participants, encrypted and in order (Ding, 2018).

\subsection{The Core Connotation of Blockchain Technology}

Blockchain technology has the subversive characteristics of decentralization and trustlessness. In the real society, information transmission and transactions between people need to rely on third-party agencies or intermediaries to provide credit enhancement measures to ensure the smooth progress of transactions. For example, when individuals buy and sell real estate, they can realize smooth transactions through real estate intermediaries and bank fund custody. However, the fees charged by the intermediary increase the cost of the transaction, and once the intermediary has problems, both parties to the transaction need 
to bear the corresponding risks and losses (Dang \& Wang, 2020). However, the blockchain can complete the signing of the contract and the transaction of funds in a point-to-point manner. Disputes on any node in the process of writing, inputting, determining, and implementing transactions by the participants will not affect the overall operation of the system and can be realized. In the true sense of the operating mode of "no middlemen earning the price difference", separation from third-party intermediaries can reduce transaction costs and risks.

\subsection{Characteristics of Blockchain Technology}

Blockchain is defined in two dimensions, narrow and broad. However, whether it is a narrow or a broad blockchain, it has the following eye-catching features: The broad blockchain technology is based on a "distributed ledger" model, With the concept of "decentralization", and the narrowly defined blockchain technology is characterized by data "non-tamperable" and "non-forgeable" (Hua, 2018). The records of the ledger are collectively recorded and maintained by all participants, and are not controlled by a centralized theme, so it has the characteristic of not being tampered with. At the same time, the blockchain data structure storage will record all historical data in time series, and each piece of data has time irreversibility and data traceability. If the data of a certain node is tampered with, the backup information of other nodes will be traced back to the historical data records, and the historical data with the most occurrences shall prevail. Therefore, counterfeiting and tampering with data will cost a huge cost and extremely difficult in the operation mode of the blockchain, thereby ensuring the security and stability of the blockchain data (Huang, Liang, Zhang, \& Wei, 2019).

\section{Analysis of the Positive Effects of Copyright Insurance}

Based on the previous theoretical analysis, this part of this article mainly analyzes the positive impact of copyright insurance, including: reducing the transaction cost of online copyright, strengthening the centralized management of online copyright, stimulating online creation, and prospering online culture. The specific analysis is as follows ( $\mathrm{Mu}, 2019)$.

\subsection{Reduce the Transaction Cost of Online Copyright}

Copyright insurance is a kind of commercial insurance with property rights as the object of insurance. The insured amount is determined by both the insurer and the insured in the form of the copyright value agreed in the contract. The insurant pays the insurance premium to the insurer. In the event of a contractual situation, the insurer an insurance method that assumes liability for the copyright insured. As a system different from "ownership", copyright must on the one hand encourage the creation and research and development of works, on the other hand, it must also realize the economic interests of copyright owners in 
copyright transactions. Coase, the originator of New Institutional Economics, believes that "clarifying property rights is a necessary prerequisite for market transactions, and clarifying property rights can reduce transaction costs and reduce the risks of rights transactions." As a collection and distribution center of copyright works, the Internet stores many unclear or flawed copyrights. Works, thereby increasing the uncertainty of copyright transactions and increasing transaction costs. Copyright insurance can underwrite the economic benefits of copyright, use the "law of large numbers" of insurance to diversify transaction risks, reduce transaction costs, promote fair competition, further ensure the stability and certainty of transactions, and promote the circulation of copyrighted goods (Sun, 2018).

\subsection{Copyright Insurance Is the Centralized Management of Network Copyright}

Some insurance companies in the United States and the European Union started intellectual property insurance business as early as the 1980s, with business scope all over the world, and better resolved patent litigation and enforcement issues. With the popularization and development of the Internet in this century, works have seen unprecedented dissemination convenience. Internet infringement has broken the traditional pattern of copyright protection and has created new challenges to laws and technologies. In 2010, Righthaven of the United States saw the business opportunity of online copyright and became the first professional company to concentrate on handling online copyright infringement litigation. Although Righthaven ended in failure, the way in its business model to concentrate copyright on its own trusteeship has reference and reference significance. Copyright insurance is actually to concentrate copyright on the insurer, and the insurer will centrally manage and form a scale effect to deal with online copyright infringement incidents with more professional and powerful means. American scholar Evan believes that the centralized handling of online copyright infringements by the insurer will reduce the occurrence of litigation and obtain compensation due to its strong team and status (Wang, 2016).

\subsection{Contribute to Stimulating Creation and Prospering Network Culture}

There are many forms of online copyright, such as uploading works to the Internet to form the right of information network dissemination; direct creation on the Internet to form original copyright. The Internet has created a variety of copyright forms and provided the most convenient means of communication. At the same time, it has also become a high incidence of infringement. Original works are quickly copied on the Internet, and network service providers use the "safe haven" principle to avoid responsibility, which has curbed creative passion to a certain extent. Due to the characteristics of its insurance system, copyright insurance can compensate copyright owners in a timely manner after infringements occur, and then redistribute economic benefits through the "risk disper- 
sion" function of insurance, thus reflecting Locke's "property rights labor value theory" to copyright owners, motivating effect. It is precisely because of the incentive effect of copyright insurance that more copyright owners will insure their own works, so that they can break away from complicated cases, concentrate on creating more and better original works, and improve the overall culture of the people. Well-being, increase the benefits of network copyright, and further prosper the network culture (Wang, 2019).

\section{Analysis of the Implementation Path of Copyright Insurance Based on Blockchain}

Technical features such as "decentralization", "distributed ledger", "non tamperable", and "non-forgeable" based on the blockchain have brought opportunities for the development of copyright insurance. Traditional copyright insurance has problems such as difficulty in confirming rights, high costs, and difficulty in pricing. However, in recent years, a number of blockchain copyright platforms have emerged in the application of blockchain in copyright. In 2017, "China Copyright Chain Smart Safe 4.0" opened a new chapter in the country to promote the application of blockchain in the copyright field. On September 3, 2018, Huiju.com shockedly released the country's first blockchain copyright registration certificate, officially opening a new era of digital copyright and blockchain technology, which also provides an unprecedented opportunity for copyright insurance (Wang \& Chen, 2019).

\subsection{Specific Measures Taken by Blockchain to Solve the Dilemma of Copyright Insurance}

\section{1) Copyright registration management}

The online platform lacks a unified copyright management platform. Literary works, videos, pictures and other works are uploaded and displayed by various channels by different authors, and works with pseudonyms, pseudonyms, and even unsigned works abound (Wang \& Zhen, 2018). However, the prerequisite for copyright transactions is to determine the ownership of the work, and copyright insurance also needs to clarify the economic interests of the insured. With the support of blockchain technology, once the copyrighted work is uploaded to the network for the first time, the hash algorithm SHA256 of the blockchain will enter a piece of information, giving the work a 256-bit hash value that cannot be tampered with (Xu, Tian, \& Li, 2017). The same work has the same Hash value. According to this, the copyright owner can upload the work to the blockchain for registration to form a unique hash value and ensure that the work is not tampered with or forged. The registration and management of copyright can use the hash algorithm to mark the work applied for registration with a unique identification, thereby playing the role of registration and publicity. At the same time, the blockchain will form a chain data structure in a sequential connection of data blocks in chronological order, so that copyright transactions are recorded in each data block in chronological order to form transaction records. This is 
equivalent to stamping a "time stamp" on copyrighted works, solving the ownership problem of "whoever creates first, who applies first, who owns the copyright" (Yao, 2013).

2) Risk pricing of copyright insurance

Copyright insurance uses the market price or transaction price of copyright as the basis for risk pricing to determine the premium amount. However, due to the inactivity of offline copyright transactions and the disadvantages of inaccurate and insufficient transaction data, it is difficult to choose a suitable reference object to determine the price; and the income method $P=S(T=1,2, \ldots, T) M t /(1$ $+r) t$, because there is no scientific basis for the selection of the parameters $\mathrm{Mt}$ (annual income brought by the work in year $T$ ) and $r$ (discount rate), which leads to great disparity in the evaluation results of various evaluation agencies, which is not of reference significance. However, the characteristics of "decentralization", "traceability" and "non-tampering" of the blockchain can realize the scientific and accurate risk pricing. Its advantages are that, first, "decentralization" and "feasibility" The feature of "traceability" breaks the information monopoly barriers of traditional information intermediaries and realizes the sharing of database resources. The insurer can obtain the copyright transaction price through the authorization agreement and distribution rules set by the copyright owner; and obtain the customer's identity information, credit information, insurance information, and compensation information for infringement cases by collecting various information on each network node. Insurers can use a huge database to model and train risk pricing, and even use machine learning to form an artificial intelligence pricing mechanism; second, the "non-tamperable" feature ensures the transparency and credibility of the data, All kinds of information no longer rely on a third party for verification, and a "self-certified" mode of data is formed through verification of more than $51 \%$ of network nodes. The authenticity and accuracy of the data is beyond doubt, providing a solid and reliable security foundation and a transparent and credible network environment for the development of Internet insurance business.

3) Automatic execution of insurance claims

There are two types of copyright insurance. The first is copyright infringement liability insurance. This type of insurance is set up by copyright users to avoid the risk of infringing on the copyright of others when the ownership is unclear. It is also called "Copyright defensive insurance". The second type of insurance is copyright enforcement insurance (Zhang \& Li, 2019). This type of insurance is set up for the copyright owner to actively request the infringer to claim compensation, so it is also called "copyright offensive insurance." In order to reflect the compensation function of copyright insurance, the insured object of "copyright offensive insurance" can not only include attorney fees, litigation fees and other claims costs, but can also be extended to the copyright owner's loss. Traditional copyright insurance has the difficulties of underwriting, claim settlement, and recovery, but the smart contract in the blockchain can solve the enforcement problem of copyright insurance (Zhang \& Dong, 2020). 
Smart contract is a concept proposed by cryptographer Nick Saab in 1995. It is a type of contract that combines contract and computer calculations and is automatically executed by a computer. Transactions in the blockchain are divided into creating smart contracts and generating information calls through the deployed smart contract interfaces. Among them, the creation of a smart contract can seal the copyright insurance claims conditions in the blockchain in the form of codes, set a number of trigger conditions, and synchronize them to the blockchain after verification by the blockchain network nodes. "Copyright defensive insurance" can set "court judgment or arbitration award insured to compensate copyright owner's loss" as the trigger condition. "Copyright Offensive Insurance" can set "Works with the same Hash value to be spread without authorization" as the trigger condition. After the smart contract is created, the network monitors the deployed contract in real time, and dynamically monitors external data to determine whether the contract preset conditions are met. When the external data meets the execution conditions after verification, the smart contract calls the interface to trigger execution. In insurance claims, if the actual compensation amount is inconsistent with the maximum insurance claim amount, the "lower of the two" rule will trigger execution, so that insurance claims are automated from creation to execution (Zhai, Chen, \& Wang, 2020).

4) The insurer exercises the right of subrogation

The biggest advantage of the insurance system is that the insurer can request compensation from a third party on behalf of the insured after paying the insured, which is also known as the insurer's right of subrogation. When exercising the right of subrogation, the insurer needs to prove that the third party does infringement and caused the copyright owner's loss, which requires the depository function of the blockchain. The evidence effect of the information on the block chain has basically been recognized by the theoretical and judicial circles. Its evidence storage function is mainly to use the "time stamp", "non-tamperable", "unforgeable" and other characteristics of the block chain. So that the information on the blockchain has undoubted credibility and proof effectiveness. In December 2018, the first case accepted by the Beijing Internet Court after its opening, "Douyin Short Video" v. "Party Video", adopted blockchain forensics technology, giving the information on the blockchain the effect of evidence. The insurer can use the technical characteristics of the blockchain to put pressure on the infringer, and use the professional and technical advantages to force the infringer to compensate the insurer and the copyright owner. In addition, at a time when intellectual property as a national strategy is hotly debated, punitive damages for intellectual property can also be introduced into copyright insurance. After obtaining the authorization of the copyright owner, the insurer can claim compensation from the infringer as an agent and the insurer, and the amount of compensation can be subject to a punitive compensation mechanism, which reflects the legal protection of online copyright. The insurer can participate in the distribution of punitive damages. On the one hand, this insurance model can punish online infringers and curb the spread of online infringements; on the 
other hand, it can also increase the income of insurers and encourage insurers to actively participate in copyright insurance. At the same time, it can also allow copyright owners to obtain more compensation (Zhou, 2021).

\subsection{Module Construction of Copyright Insurance Platform Based on Blockchain}

1) Registration and registration module

a) Registration

On January 10, 2019, the National Internet Information Office announced the "Regulations on the Management of Blockchain Information Services". According to Article 8 of the law, the insured person needs to upload his ID card, business license, and mobile phone For information such as number, the platform needs to verify and authenticate the true identity of the insured, and at the same time, it needs to verify that the insured is a qualified civil subject and has the qualifications to sign an insurance agreement.

b) Registration

The insured shall register the digital copyright of the work that he needs to underwrite on the blockchain and sign the CRC contract (Copyright Registration Contract) on the blockchain. After the digital copyright is successfully registered, the CRC contract will extract the Hash The value is used as the DCI (Digital Copyright Identifier, DCI) of the work. In order to unify the labeling of works on different blockchain platforms, in March 2018, the China Copyright Protection Center released the "DCI System Industrial Application White Paper", proposing strategic measures to accelerate the construction of the Digital Copyright Unique Identifier (DCI) system.

2) Copyright insurance contract module

Insurance contracts belong to the category of contract law. In order to reach the unanimous agreement of the insurer and the insured, the insurer needs to display the content of the format clauses on the platform in text form, and the insurance exemption clauses should be prominently indicated to the insured. The insurer signs the electronic contract by clicking on the mouse to "read the text of the contract and agree to sign". This information will be verified by the network node and synchronized to the blockchain, and a smart contract will be created. The smart contract will record contract matters in computer language, set trigger conditions in computer code, and run on multiple network nodes (Zhang, 2019).

3) Automatic execution module for insurance claims

Blockchain can realize infringement monitoring on the entire network platform, using hash value tracking technology to detect the reprinting of works, which allows each certified work to feed back on its entire network transmission path in real time, including formal authorized transmission and non-formal authorized transmission. And it can automatically solidify and save infringement evidence, form an infringement report and notify the copyright owner in time, 
so that the copyright owner can fulfill the obligation of "notify-delete" and safeguard rights in a timely manner. In "copyright offensive insurance", when the network node verifies that "the same hash value work is spread without authorization", the smart contract will trigger the automatic execution of insurance claims. In "copyright defense insurance", the blockchain platform can be connected to the Internet court system. Once the court decides that the insured loses the lawsuit, the smart contract will trigger the automatic execution of insurance claims, and the insurer will follow the "judgment of compensation and insurance Claims are automatically settled based on the rule of the lower of the maximum claim amount".

\section{The Challenges Still Faced by Blockchain-Based Copyright Insurance}

To sum up, blockchain-based copyright insurance has a broad market space and operating space, but technology brings development opportunities and also challenges, which need to be resolved in future development.

\subsection{Moral and Legal Risks}

As a scientific and technological means, blockchain can only reduce operational risks, but cannot prevent moral and legal risks. After all, computer code and language are written by humans, and the trigger conditions set are also based on human wisdom, based on blockchain there is also the problem of fraudulent insurance in the field of copyright insurance. Take "copyright defensive insurance" as an example. If the insured colludes with a third party to create false litigation or deliberately exaggerate the amount of loss, the court will rule that the insured loses the lawsuit, which triggers insurance claims. In this case, the smart contract of the blockchain will be automatically executed in accordance with the pre-set procedures. It cannot judge the authenticity of the disputed facts in the court judgment, nor can it verify whether the insured and the third party are malicious outside the network. Collusion, so blockchain cannot solve moral and legal risks.

\subsection{Avoid the Identification of Infringement through Technical Means}

The blockchain uses the technical means of tracking the hash value of works to realize the monitoring of the entire network. Works with the same hash value will be recognized as the same work, and then it will be judged whether it has a formal authorization to spread. However, if the content of a work is not substantively changed and does not meet the "creative" standard, it will not be recognized as a new work in accordance with the provisions of the Copyright Law. However, blockchain technology cannot realize the judgment standard of artificial intelligence. Once the content of the work is changed, it will be given a new Hash value to distinguish it from the original copyrighted work independently, thereby avoiding the monitoring and review of the blockchain. With the further 
development and application of artificial intelligence and neural networks, artificial intelligence review methods can be applied in the blockchain to achieve technological breakthroughs.

\subsection{Security Issues}

The blockchain adopts asymmetric encryption for information interaction. The public key and the private key have a strong match. The content encrypted by the public key can only be decrypted by the corresponding private key; the content encrypted by the private key can only be decrypted by the corresponding public key. The key can be decrypted. In blockchain applications, the user's account is the public key, and the means of information interaction is the private key. Once the private key is lost, it cannot be decrypted. There is no center to reset the private key, which causes security risks. In addition, in the information verification process, more than $51 \%$ of the network nodes need to be verified before being incorporated into the blockchain. However, the integration of computing power and other technical means may pose a security threat to the application of the blockchain.

\subsection{Misjudgment of "Fair Use"}

As a restriction on copyright, "fair use" has been generally recognized by the copyright laws of various countries. As a form of copyright, online copyright is naturally restricted by "fair use". "Fair use" ensures freedom of the press and citizens' freedom of consultation, and also promotes the dissemination of knowledge in the education industry, maintaining "rational fairness and justice." However, the blockchain technology cannot identify the purpose of using the work, causing "fair use" to be labeled as infringement. Therefore, in the monitoring of "unauthorized dissemination" of the blockchain, it is necessary to combine the industry nature, media type, work type and other factors of the work user to make a comprehensive judgment to avoid the mechanical nature of the technology that affects the copyright of "fair use".

\section{Suggestions for the Future Development of Copyright Insurance Based on Blockchain}

Based on the previous analysis of copyright insurance value and development dilemma, this part of the article focuses on the future development of blockchain-based copyright insurance, including: First, the regulatory authorities must strengthen multi-party collaboration to form standardized copyright insurance technical standards. Unify and coordinate the development of copyright insurance; second, vigorously support the development of blockchain technology, and promote the wide application of blockchain technology; third, the ownership certificate, the blockchain technology will write the intended use of copyright, allowing everyone to real-time Track users who use their works, help their users confirm authorization opportunities, and optimize the commercial value 
of works; fourth, promote non-standard smart contracts.

\subsection{Multi-Party Collaboration}

Through the National Intellectual Property Office, Intellectual Property Protection Association, and Intellectual Property Law Firms, multiple parties use blockchain distributed accounting technologies to provide a copyright insurance platform to reduce the credit risk and sunk costs between intellectual property owners and users. The blockchain information implements a non-tamperable and traceable mode, and the data between related users is updated in real time and real-time accounting.

Blockchain can provide a new platform for copyright insurance, but emerging technologies cannot completely replace legal counsel and window guidance from copyright protection agencies. The owner does not have the ability to completely rely on automation or artificial intelligence to solve the ever-changing copyright issues. Intellectual property law firms should actively learn and apply the blockchain to help owners provide copyright protection in the blockchain. The State Intellectual Property Office and the Intellectual Property Protection Association should accelerate the formulation of the scope and technical standards adopted by the blockchain to protect the owner.

\subsection{Improve Blockchain Technology}

Blockchain technology has been rapidly extended to the field of copyright protection through the upsurge of Bitcoin, and governments of various countries have realized the significant impact of blockchain technology on the cultural information industry of their countries. In the practice of copyright protection applications, the development and promotion of blockchain application tools represented by Ethereum has made it possible to use copyright protection in the blockchain, and the blockchain has entered the 3.0 era. The production model of "manufacturing mining machines, mining, and speculating electronic money" consumes a large amount of power resources and electronic equipment resources in various countries. In the future, it is necessary to increase the development of token blockchain and improve copyright protection to write into the blockchain. The model enables people who need copyright protection to obtain tokens and improve the efficiency of resource use.

\subsection{Proof of Ownership}

Blockchain can provide a new model for copyright insurance. The intended use of copyright is written into the blockchain technology, allowing everyone to track users who use their works in real time, helping their users confirm authorization opportunities, and optimize the commercial value of the works. The copyright owner and related users can check the ownership chain of the work in the blockchain, including licensing, sub-licensing and transfer. The digital certificate provided by ownership can help third parties identify the authenticity of 
the work, the owner, and the scope of authorization, and help authors and users prevent infringement and fraud. Proof of ownership reduces the cost of licensing transactions and improves the efficiency of using works by creating a direct chain point-to-point transaction between the author and the user.

\subsection{Non-Standard Smart Contracts}

The existing smart contracts in the blockchain are only standard contracts, and the scope of use is relatively narrow. Smart contracts are composed of computational codes added to the blockchain, while non-standard smart contracts are mainly restricted by the automatic execution of computational codes and the implementation of contract terms. The research and development of non-standard smart contracts requires the joint research and development of the computer field and intellectual property law firms. Language programming is used to improve the ability of computing coding to identify non-standard smart contracts. Through smart contracts, external data is provided with a link entry and permanently written into the blockchain. Promote the recording of related transactions in the blockchain.

\section{Research Conclusion}

To sum up, with the rapid development of information technology and the widespread popularization of mobile Internet, various online copyright works or writings will appear quickly and have a great impact on people's production and life. However, with the rapid development of online works, a large number of infringements will restrict the healthy development of online copyright. Therefore, exploring copyright insurance has become an important measure and a useful exploration to resolve network copyright protection. By virtue of its compensation function and system advantages, copyright insurance can have a positive effect on online copyright. The author believes that, thanks to the rapid development and wide application of blockchain technology, the conditions for building a copyright insurance platform module are already available. This is an important measure to enhance the effectiveness of copyright insurance and can efficiently promote the development of copyright insurance. Of course, the current technical problems of building a copyright insurance platform still exist, and iterative technology must be used to resolve various problems.

\section{Conflicts of Interest}

The author declares no conflicts of interest regarding the publication of this paper.

\section{References}

Cao, Z. (2017). Study on the Coordinated Development of Copyright Insurance System and Digital Publishing Industry. China Publishing, No. 20, 67-70.

China Academy of Information and Communications Technology (2018). 2017 Annual 
Report on China's Network Copyright Protection (Abstract Edition). China Publishing, No. 9, 14-18.

Cui, M. X. (2019). Analysis of the Evidence Law Value of Blockchain Electronic Deposits-Taking Hangzhou Information Network Communication Right Dispute Case as an Example. Wireless Internet Technology, 16, 149-151.

Dang, X., \& Wang, W. Y. (2020). Research on Legal Issues Related to the Protection of Digital Music Copyright Blockchain Technology. Electronic Intellectual Property, No. 4, 28-42.

Ding, X. W. (2018). Thinking about the Integration of Blockchain into Copyright Insurance. Nanjing Social Sciences, No. 9, 120-125.

Hua, J. (2018). The Application of Blockchain Technology and Smart Contracts in the Confirmation and Transaction of Intellectual Property Rights and Its Legal Regulations. Intellectual Property, No. 2, 13-19.

Huang, Y. X., Liang, Z. H., Zhang, M. D., \& Wei, B. (2019). Blockchain Learning Achievement Management and Control Model for Credit Bank. Computer Engineering, 45, 18-24.

McAlpine, E. (2013). Of Oatmeal, Bears, and NPEs: Ensuring Fair, Effective, and Affordable Copyright Enforcement through Copyright Insurance. American University Intellectual Property Brief, 19, 34.

Mu, X. M. (2019). New Ideas for Digital Copyright Protection Based on Blockchain Technology-Comment on "2018 China Network Copyright Protection Annual Report”. Publishing Wide Angle, No. 19, 91-93.

Sun, J. (2018). Blockchain Encyclopedia: An Introductory Manual for Bitcoin and Other Digital Currencies That Everyone Can Understand (p. 6). Electronics Industry Press.

Wang, H. (2016). Blockchain Technology and Internet Insurance. China Finance, No. 10, 74-76.

Wang, H. C. (2019). The Progress and Challenges of Blockchain in the Application of Digital Rights Management Platform. Editor's Friends, No. 10, 80-86.

Wang, Q., \& Chen, X. T. (2019). The Application and Legal Regulation of Blockchain Technology in Digital Copyright Protection. Journal of Hubei University (Philosophy and Social Sciences Edition), 46, 150-157.

Wang, W. L., \& Zhen, Y. (2018). The Application of Blockchain Technology in the Financing of Digital Publishing Supply Chain. Publishing and Distribution Research, No. $8,31-34$.

Xu, M. X., Tian, Y., \& Li, J. Y. (2017). Illustrated Blockchain (p. 6). CITIC Publishing House.

Yao, Y. (2013). On the Perfection of My Country's Copyright Transaction Guarantee Insurance (p. 28). Liaoning University.

Zhai, S. P., Chen, S. J., \& Wang, Y. J. (2020). Research on the Model of Digital Copyright Deposit System Based on Blockchain. Computer Engineering and Applications, 56, 13-21.

Zhang, B. Q., \& Li, L. (2019). Digital Copyright Benefit Balance Based on Blockchain Technology. China Publishing, No. 11, 22-25.

Zhang, S., \& Dong, Y. (2020). Research on Digital Copyright Protection Based on Blockchain Technology. Science and Technology Management Research, 40, 132-136.

Zhang, Y. H. (2019). Blockchain and Digital Publishing: Mechanism, Prospects and Challenges. People's Forum, No. 33, 76-77.

Zhang, Y., \& Liang, Y. D. (2017). Research on Decentralized Digital Publishing Platform 
Based on Blockchain Technology. Publishing Science, 25, 13-18.

Zhou, L. (2021). Research on the Balance Strategy of Public Library Digital Copyright Transactions from the Perspective of Blockchain. Library Work and Research, No. 1, 73-77. 